\title{
Genetic Variant Score and Arrhythmogenic Right Ventricular Cardiomyopathy Phenotype in Plakophilin-2 Mutation Carriers
}

\author{
Anneli Svensson $^{a}$ Pyotr G. Platonov ${ }^{b}$ Kristina H. Haugaac Wojciech Zareba ${ }^{\text {d }}$ \\ Henrik Kjærulf Jensen ${ }^{\mathrm{e}}$ Henning Bundgaard ${ }^{f}$ Thomas Gilljam $^{g}$ Trine Madsen $^{\mathrm{h}}$ \\ Jim Hansen ${ }^{i}$ Lars A. Dejgaard ${ }^{c}$ Lars O. Karlsson ${ }^{a}$ Anna Gréen ${ }^{j}$ \\ Bronislava Polonsky ${ }^{d}$ Thor Edvardsen ${ }^{c}$ Jesper Hastrup Svendsen ${ }^{k}$ \\ Cecilia Gunnarsson ${ }^{\mathrm{j}, \mathrm{I}}$ \\ aDepartment of Cardiology and Department of Health, Medicine and Caring Sciences, Linköping University, \\ Linköping, Sweden; ${ }^{b}$ Department of Cardiology, Clinical Sciences, Lund University and Arrhythmia Clinic, Skåne \\ University Hospital, Lund, Sweden; 'Department of Cardiology, Centre for Cardiological Innovation, Oslo University \\ Hospital, Oslo, Norway and University of Oslo, Oslo, Norway; 'University of Rochester Medical Center, Rochester, \\ NY, USA; 'Department of Cardiology, Aarhus University Hospital and Department of Clinical Medicine, Aarhus \\ University, Aarhus, Denmark; fUnit for Inherited Cardiac Diseases, The Heart Center, The National University \\ Hospital, Copenhagen, Denmark; ${ }^{9}$ Department of Cardiology, Institute of Medicine at Sahlgrenska Academy, \\ University of Gothenburg, Gothenburg, Sweden; ' Department of Cardiology, Aalborg University Hospital, Aalborg, \\ Denmark; 'Department of Cardiology, Herlev-Gentofte Hospital, University of Copenhagen, Hellerup, Denmark; \\ 'Department of Clinical Genetics, and Department of Biomedical and Clinical Sciences, Linköping University, \\ Linköping, Sweden; 'Department of Cardiology, The Heart Centre, Rigshospitalet, University of Copenhagen, \\ and Danish National Research Foundation Centre for Cardiac Arrhythmia (DARC), Copenhagen, Denmark and \\ Department of Clinical Medicine, Faculty of Health and Medical Sciences, University of Copenhagen, Copenhagen, \\ Denmark; 'Centre for Rare Diseases in Southeast Region of Sweden, Linköping University, Linköping, Sweden
}

\section{Keywords \\ Arrhythmogenic right ventricular cardiomyopathy . Combined Annotation Dependent Depletion score · Plakophilin-2 · Arrhythmia · Ventricular tachycardia}

\begin{abstract}
Introduction: Whether detailed genetic information contributes to risk stratification of patients with arrhythmogenic right ventricular cardiomyopathy (ARVC) remains uncertain. Pathogenic genetic variants in some genes seem to carry a higher risk for arrhythmia and earlier disease onset than others, but comparisons between variants in the same gene
\end{abstract}

karger@karger.com www.karger.com/crd

Karger $\stackrel{\text { ' }}{5}$

GOPEN ACCESS
(C) 2021 The Author(s)

Published by S. Karger AG, Basel

This article is licensed under the Creative Commons Attribution 4.0 International License (CC BY) (http://www.karger.com/Services/ OpenAccessLicense). Usage, derivative works and distribution are permitted provided that proper credit is given to the author and the original publisher. have not been done. Combined Annotation Dependent Depletion (CADD) score is a bioinformatics tool that measures the pathogenicity of each genetic variant. We hypothesized that a higher CADD score is associated with arrhythmic events and earlier age at ARVC manifestations in individuals carrying pathogenic or likely pathogenic genetic variants in plakophilin-2 (PKP2). Methods: CADD scores were calculated using the data from pooled Scandinavian and North American ARVC cohorts, and their association with cardiac events defined as ventricular tachycardia/ventricular fibrillation (VT/VF) or syncope and age at definite ARVC diagnosis were

Anneli Svensson and Pyotr G. Platonov contributed equally.
Correspondence to:

Anneli Svensson, anneli.svensson@ regionostergotland.se 
assessed. Results: In total, 33 unique genetic variants were reported in 179 patients ( 90 males, 71 probands, 96 with definite ARVC diagnosis at a median age of 35 years). Cardiac events were reported in 76 individuals (43\%), of whom 53 had sustained VT/VF (35\%). The CADD score was neither associated with age at cardiac events (HR 1.002, 95\% Cl: $0.953-$ $1.054, p=0.933$ ) nor with age at definite ARVC diagnosis (HR $0.992,95 \%$ Cl: $0.947-1.039, p=0.731)$. Conclusion: No correlation was found between CADD scores and clinical manifestations of ARVC, indicating that the score has no additional risk stratification value among carriers of pathogenic or likely pathogenic PKP2 genetic variants.

(C) 2021 The Author(s)

Published by S. Karger AG, Basel

\section{Introduction}

Arrhythmogenic right ventricular cardiomyopathy (ARVC) is a heritable heart muscle disease involving primarily the right ventricle (RV). A disease-causing genetic variant is identified in $40-60 \%$ of probands [1] and is predominantly related to one of the genes coding for desmosomal proteins, of which plakophilin-2 (PKP2) is the most commonly involved [2-5]. ARVC is a progressive disease with large differences in clinical presentation, including ventricular arrhythmias and heart failure, and the diagnosis is based on Task Force Criteria (TFC) proposed in 1994 and modified in 2010 (TFC2010) [6]. Ventricular arrhythmias and sudden cardiac death (SCD) may be the first symptoms [7].

The evidence of early involvement also of the left ventricle in many cases, and sometimes only here, has led to the term "arrhythmogenic cardiomyopathy" (AC or ACM). For this study, we used data from registries evaluating individuals using TFC2010 criteria and have hence kept the term ARVC.

A challenging part of care for patients with ARVC and their families is the counseling regarding the age of disease manifestation in gene variant carriers and the risk of ventricular arrhythmias and SCD [8-11]. Genetic findings may add further knowledge in this area. No convincing evidence of the use of genetic information for risk stratification has been reported for single PKP2 genetic variants, despite being the most frequent finding. More than 1 pathogenic desmosomal genetic variant in the same individual however seems to be associated with higher arrhythmia risk and earlier progress of the disease [12].

In clinical practice, genetic variants are classified according to the American College of Medical Genetics and
Genomics (ACMG) and Association for Molecular Pathology (AMP) guidelines [13]. These guidelines recommend using specific standard terminology for genetic variants in 5 categories: pathogenic, likely pathogenic, uncertain significance, likely benign, and benign. The Combined Annotation Dependent Depletion (CADD) score provides a framework for estimating the relative risk of human genetic variants, and it correlates with both molecular functionality and pathogenicity [14]. The CADD score reflects the difference between the characteristics of genetic variation that is tolerated in the human genome and the characteristics of pathogenic variants, and its predictions are based on a logistic regression model that considers evolutionary conservation, regulatory and transcript information, and protein-level scores. The CADD score integrates 63 different annotations into a single, quantitative score and has been used in the laboratory and previous studies $[15,16]$ to identify possible pathogenic variants.

However, since the CADD score is not a measure of certainty on whether a genetic variant is pathogenic or not but rather a measure of dysfunctionality on a protein level associated with a specific variant, it appears plausible that this dysfunctionality can be related to the degree of disease manifestations and age at disease penetrance. We have found no study regarding this specific question published so far. The current study intended to investigate, in a cohort of ARVC patients carrying PKP2 variants, whether the CADD score is associated with clinical manifestations of the disease, including the risk of ventricular arrhythmias.

\section{Methods}

The study group is a pooled cohort recruited in 2 prospective observational ARVC registries: the Nordic ARVC Registry and the North American Multidisciplinary Study of ARVC. The Nordic ARVC Register (www.arvc.dk) is an observational register-based study initiated in 2010 that includes patients with definite ARVC by 2010 TFC [6] and their genotype-positive family members enrolled from 9 centers in Denmark, Norway, and Sweden [17]. The North American Multidisciplinary Study of ARVC is also a multicenter study, prospectively gathering information on clinical outcomes, diagnostic measures, and genetics in ARVC probands and their family members [18].

For this study, only individuals carrying a single variant of $P K P 2$ that was considered pathogenic or likely pathogenic at the time of evaluation were included. The genetic analyses were performed at the discretion of each participating center using the techniques available at the time of evaluation. Even though majority of study participants were genetically assessed recently when NGS technology became available, there are patients who were examined at the 


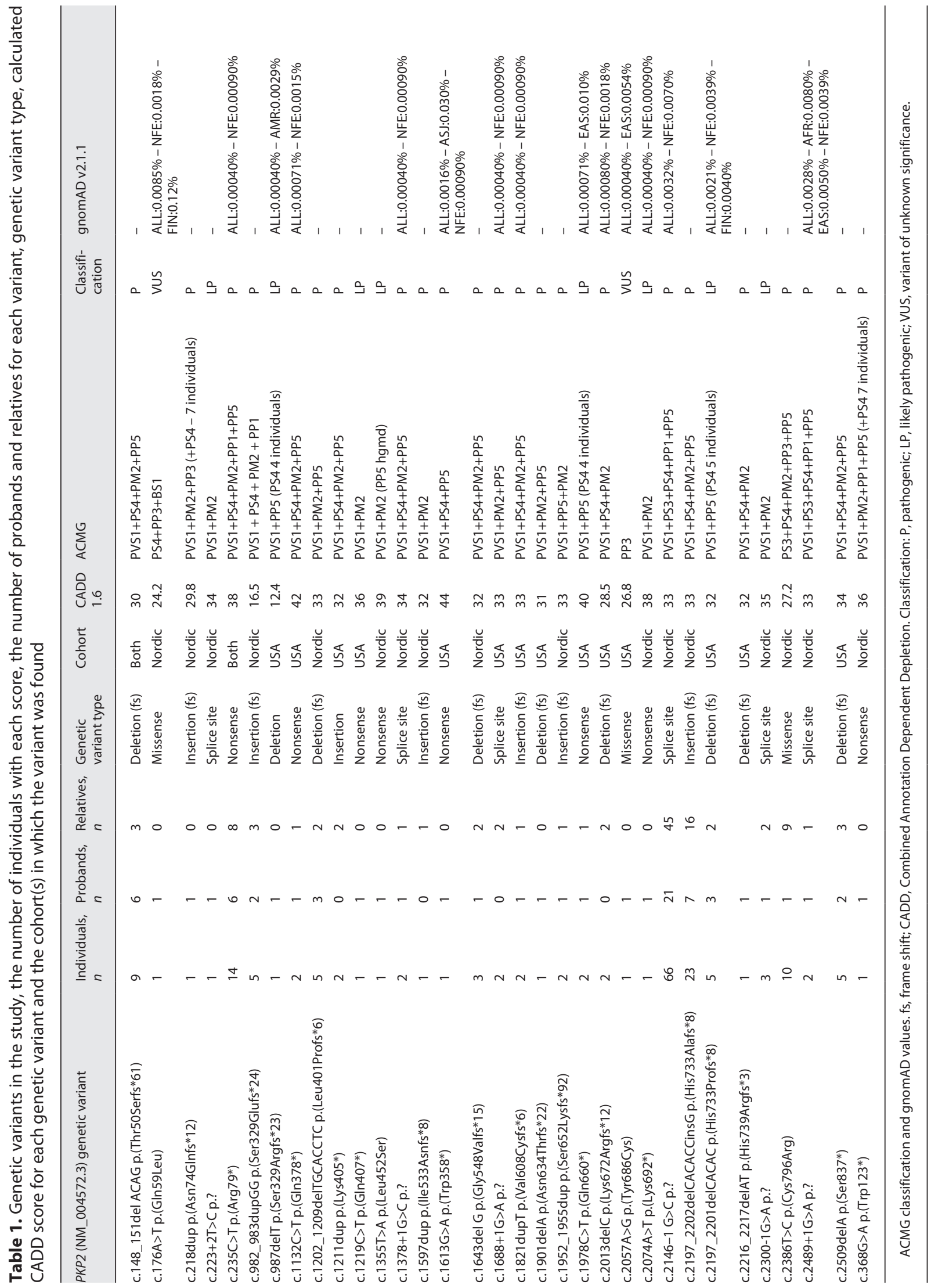


Table 2. Demographic and clinical characteristics of $P K P 2$ carriers at time of last evaluation ( $p$ values comparing participants from Nordic ARVC registry to participants from the North American Multidisciplinary ARVC Study)

\begin{tabular}{|c|c|c|c|c|}
\hline & All & Nordic & USA & $p$ value \\
\hline$N$ & 179 & 144 & 35 & \\
\hline CADD, median (IQR) & $33.0(33.0-33.0)$ & $33.0(33.0-33.0)$ & $33.0(32.0-38.0)$ & 0.175 \\
\hline Probands, $n(\%)$ & $71(40)$ & $52(36)$ & $19(54)$ & $0.049^{\dagger}$ \\
\hline Age at ARVC diagnosis, median (IQR) & $35(24-51)$ & $35(26-53)$ & $31(22-49)$ & 0.417 \\
\hline Age at enrollment, median (IQR) & $37(24-51)$ & $37(24-52)$ & $37(24-50)$ & 0.748 \\
\hline ICD carrier, $n(\%)$ & $82(46)$ & $59(41)$ & $23(66)$ & $0.012^{+}$ \\
\hline Treatment with beta blocker, $n(\%)$ & $66(37)$ & $46(32)$ & $20(57)$ & $0.006^{+}$ \\
\hline Treatment with antiarrhythmic drug(s), $n$ (\%) & $50(28)$ & $39(27)$ & $11(31)$ & 0.608 \\
\hline VT ablation performed, $n(\%)$ & $19(11)$ & $14(10)$ & $5(14)$ & 0.539 \\
\hline Definite ARVC diagnosis according to TFC 2010, $n(\%)$ & $96(54)$ & $73(51)$ & $23(66)$ & 0.111 \\
\hline \multicolumn{5}{|l|}{ Study endpoints, $n(\%)$} \\
\hline Aborted sudden death & $12(7)$ & $10(7)$ & $2(6)$ & 1.000 \\
\hline Syncope & $18(10)$ & $12(8)$ & $6(17)$ & 0.126 \\
\hline Years of follow-up, median (IQR) & $5.6(3.3-8.0)$ & $6.3(4.0-9.0)$ & $3.1(2.1-4.1)$ & $0.000^{\dagger}$ \\
\hline
\end{tabular}

Tested with the Mann-Whitney $U$ test (clinical characteristics and outcomes) or Fisher's exact test (frequency of events), significant $\left({ }^{\dagger}\right)$ if $p<0.05$. ARVC, arrhythmogenic right ventricular cardiomyopathy; CADD, Combined Annotation Dependent Depletion; TFC, Task Force Criteria; SCD, sudden cardiac death; VT, ventricular tachycardia; VF, ventricular fibrillation; ATP, anti-tachycardia pacing; IQR, interquartile range. * Syncope, appropriate ICD therapy (ATP or shock), aborted SCD.

time when the panels were limited to desmosomal genes sets focused on PKP2, DSG2, DSC2, DSP, and JUP as a standard. The genetic variants reported were for this work classified using ACMG guidelines (American College of Medical Genetics and Genomics) [13]. Those patients who were genotyped before the ACMG guidelines were introduced were re-classified accordingly using the description of the reported genetic variant (Table 1).

The CADD score was calculated using the online calculation provided by the University of Washington and HudsonAlpha Institute for Biotechnology, available at http://cadd.gs.washington. edu/ (version GrCh 37-v1.6). The CADD score calculated for each genetic variant was analyzed for association with study endpoints. For this analysis, we used PHRED-scaled CADD scores as this approach is recommended by the algorithm developers [14].

Baseline clinical and demographic characteristics as well as diagnostic information required by the updated TFC from 2010 [6] were collected. The 2010 Task Force diagnostic criteria were assessed at the time of diagnosis in those who fulfilled the definite ARVC diagnosis requirements or at the last available follow-up in genotype-positive individuals who did not reach the "definite" diagnostic category. Clinical endpoints of interest were (1) sustained ventricular tachycardia (VT) or ventricular fibrillation (VF) defined as either ECG-documented ventricular arrhythmia, appropriate ICD therapy (anti-tachycardia pacing or shock), SCD, or aborted SCD; (2) the first cardiac event defined as syncope or any VT/VF; and (3) the age at definite ARVC diagnosis by the TFC. Patients were censored at the time of the last follow-up.
The Mann-Whitney test was used to compare clinical characteristics and outcomes depending on the CADD score, while Fisher's exact test was used to compare the frequency of events. Receiver operating characteristic curve analysis was performed to assess the relationship between the CADD score and cardiac events, VT/VF, and fulfillment of diagnostic criteria. Data are presented as median and interquartile range (IQR), unless stated otherwise. A $p$ value $<0.05$ is considered significant. Statistical analysis was performed with SPSS version 25.0 (IBM SPSS Statistics for Windows, version 25.0. Armonk, NY, USA: IBM Corp).

\section{Results}

A total of 179 individuals were identified in the registries as carriers of a pathogenic or likely pathogenic genetic variant in the PKP2 gene and were included in the study, none of them carrying any other known pathogenic genetic variant in other ARVC-related genes. Table 2 presents clinical characteristics of the study cohort. Vast majority of patients were recruited in Scandinavia $(n=144)$, of whom 99 carried one of the 3 most common genetic variants (Table 1 ). 
Table 3. 2010 Task Force diagnostic criteria in the cohort, prevalence in all individuals and comparison between the 2 participating registries

\begin{tabular}{|c|c|c|c|c|}
\hline & All, $n=179$ & Nordic, $n=144$ & USA, $n=35$ & $p$ value \\
\hline \multicolumn{5}{|l|}{ Imaging, $n(\%)$} \\
\hline Major & $64(36)$ & $49(34)$ & $15(43)$ & 0.330 \\
\hline Minor & $9(5)$ & $7(5)$ & $2(6)$ & 0.836 \\
\hline \multicolumn{5}{|l|}{ Tissue, $n(\%)$} \\
\hline Biopsy performed & $30(17)$ & $18(13)$ & $12(34)$ & \\
\hline Major* & $4(13)$ & $1(6)$ & $3(25)$ & 0.131 \\
\hline Minor* & $1(3)$ & $0(0)$ & $1(8)$ & 0.221 \\
\hline \multicolumn{5}{|l|}{ Repolarization, $n$ (\%) } \\
\hline Major & $60(34)$ & $47(33)$ & $13(37)$ & 0.614 \\
\hline Minor & $81(45)$ & $64(44)$ & $17(49)$ & 0.661 \\
\hline \multicolumn{5}{|l|}{ Depolarization, $n$ (\%) } \\
\hline Major & $7(4)$ & $6(4)$ & $1(3)$ & 0.721 \\
\hline Minor & $67(37)$ & $53(37)$ & $14(40)$ & 0.727 \\
\hline \multicolumn{5}{|l|}{ Arrhythmia, $n(\%)$} \\
\hline Major & $32(18)$ & 27 (19) & $5(14)$ & 0.538 \\
\hline Minor & $68(38)$ & $53(37)$ & $15(43)$ & 0.509 \\
\hline \multicolumn{5}{|l|}{ Family history, $n(\%)$} \\
\hline Major & $179(100)$ & $144(100)$ & $35(100)$ & 1.000 \\
\hline Minor & $7(4)$ & $2(1)$ & $5(14)$ & $0.000^{\dagger}$ \\
\hline
\end{tabular}

Tested with the Mann-Whitney $U$ test, significant $\left(^{\dagger}\right)$ if $p<0.05 .{ }^{*}$ Percentage of those who had biopsy performed.
The CADD score ranged from 12.4 to 44 . In the receiver operating characteristic curve analysis, the area under the curve was 0.53 (95\% CI $0.44-0.62, p=0.526$ ) for association of the CADD score with cardiac events, 0.50 (95\% CI $0.41-0.59, p=0.999$ ) for sustained VT/VF and 0.52 (95\% CI 0.43-0.61, $p=0.661)$ for age at ARVC diagnosis. ARVC patients from the North American cohort more often received ICD implant and beta-blocker therapy but otherwise did not show significant differences in phenotypic manifestations of the disease as compared to the Nordic participants (Table 2).

The presence of major and minor TFC 2010 diagnostic criteria is outlined in Table 3. Due to genetic variant carrier status, all individuals in the study fulfilled a major diagnostic criterion from a family history/genetics category.

Table 1 displays the list of unique $P K P 2$ genetic variants reported in the cohort, the number of individuals carrying each variant and the variants' respective CADD scores. Patients with definite ARVC did not differ in the median CADD score compared to genotype-positive family members who did not reach the definite diagnostic threshold (33.0 [IQR 32.3-33.8] vs. 33.0 [33.0-33.0], $p=$ 0.406).

The median CADD score was the same in patients who at any time developed cardiac events as in patients without cardiac events (33.0 [IQR 32.3-33.8] vs. 33.0 [IQR
33.0-33.0], $p=0.422$, and regarding the sustained VT/VF outcome (33.0 [33.0-33.0] vs. 33.0 [IQR 33.0-33.0], $p=$ $0.575)$. The 33 genetic variants are reported in Table 1 . They were splice site mutations $(n=6,18 \%)$, nonsense variants $(n=8,24 \%)$, deletions $(n=9,27 \%)$, missense variants $(n=3,9 \%)$, and insertions $(n=7,21 \%)$. Seventeen of the variants were only found in the Nordic ARVC registry, 14 were only found in the US cohort, and 2 variants were found in both registries. We did not observe any association between the genetic variant categories in the highest number of individuals (nonsense and deletion) and cardiac events, sustained VT/VF, or age at diagnosis.

\section{Discussion}

To the best of our knowledge, this is the first attempt to correlate the CADD score to the ARVC phenotype in PKP2 mutation carriers. We found no significant correlation between CADD score values and either arrhythmic events or age at ARVC diagnosis.

ARVC patients have an increased risk for ventricular arrhythmic events, including SCD [19, 20]. Several studies of genetic variants intended to use specific genetic information for risk stratification have not proven useful $[21,22]$, but the number of patients in those studies has often been limited due to relative rarity of the disease. 
Reliable estimation of gene variant pathogenicity is a major challenge in clinical genetics, underlined by the introduction of next-generation sequencing in clinical diagnostics. In 2015, the American College of Medical Genetics and Genomics (ACMG) and the Association for Molecular Pathology (AMP) proposed guidelines to classify variants in genes associated with Mendelian diseases according to their potential pathogenicity [13]. Reclassification of genetic variants in the ARVC context has been a well-appreciated phenomenon, and the initial interpretation of genetic variant may change with time in both directions. Two of the reported pathogenic genetic variants from our cohort would now be considered benign and were therefore excluded from this study. Two additional variants were at the time of inclusion in the register classified as pathogenic but would now be classified as variant of unknown significance, but not benign, and these are not excluded.

In clinical practice, there is often an issue of whether a given genetic variant is benign or pathogenic. The fairly recent introduced CADD score is described in the reference article from 2014 as a promising alternative or complementary tool [14] and that the method offers a standardized, genome-wide variant scoring metric that incorporates the weighted results of different prediction tools such as PolyPhen and SIFT and the genomic annotation sources such as ENCODE [23]. The resulting CADD score is expressed as a measure of deleteriousness for indels (an insertion or deletion of bases in the genome) and single-nucleotide variants. It is not limited to any specific type of genetic variant or organ systems. The result ranges from 0 to around 45, with no formal upper limit, and no clear cutoff for the point at which a variant is considered pathogenic. A high score represents a variant that is not stabilized by selection and is more often disease-causing than expected at random. By contrast, a low score (single digit) indicates that a variant seems to be an evolutionarily stable, commonly occurring genetic variant with no harm to an organism.

The CADD score has been used as one of several tools to sort out pathogenic variants in studies of simulated whole genomes, not as the single parameter assessing finer gradations in pathogenicity $[15,16]$. As of now, there are limited data regarding the clinical use of the scoring system [24], and there are no published studies in which the CADD score has been proven clinically useful as part of the risk stratification in heart diseases.

Genetic variants in PKP2 are known to be associated with ARVC [25-27]. Genetic variants in PKP2 may cause ARVC due to desmosomal instability and degradation
[25], but newer studies also suggest that other mechanisms, such as interference with intracellular calcium homeostasis [28], may cause ventricular arrhythmias without myocardial structural changes. In the present study, we analyzed all genetic variants reported as pathogenic or likely pathogenic in PKP2 in the registries using ACMGAMP guidelines [13]. One can speculate that a high CADD score is due to a genetic variant resulting in a more severely dysfunctional protein that may predict more arrhythmic events in ARVC, and that was our hypothesis for this study.

A recent review and meta-analysis of risk stratification in ARVC provides an overview of risk factors and their predictive potential [29]. Genotype-positive individuals more commonly present with ventricular arrhythmias at a younger age [30]. We also know that patients with pathogenic genetic variants coding for nondesmosomal proteins such as transmembrane protein 43, phospholamban, and lamin A/C seem to be at higher risk for arrhythmia than patients with desmosomal gene variants [31]. Individuals with pathogenic genetic variants in desmoglein-2 is more prone to heart failure than those with variants in $P K P 2$ [32].

The algorithm of the CADD score cannot consider the presence of $>1$ genetic variant. In the present study, 9 patients were excluded (6 from the Nordic cohort and 3 from the US cohort) because of carrying double pathogenic genetic variants, 1 in PKP2 and the other in another ARVC-related gene.

New studies continue to contribute to aiding in risk stratification [19, 33-35]. Apart from different genetic variants, there are other factors known to predispose patients to arrhythmic events, such as physical activity [36-38].

Since there is however significant variability in phenotypic expression and penetrance among individuals with the exact same gene variant $[5,39-41]$, genetic scores may be used to compare the risk between families with different genetic variants, but not between individuals in the same family. This points out the importance of re-evaluation of genetic analysis done in the past since the knowledge of genetic variants has improved during the last years.

Genetic profiles of patients recruited in the USA differed from the genetic profiles of patients recruited in the Nordic countries (Table 1). Of 33 different genetic variants found, $2(6 \%)$ were present in both the Nordic and the US cohorts, while 17 (52\%) were present only in the Nordic individuals and 14 (42\%) only in the US cohort. The differences in the use of ICDs and beta-blockers (both ICDs and beta-blockers were more frequently used 
in the US cohort) likely reflect the differences in treatment preferences between the regions, since neither the number of individuals with definite ARVC diagnosis nor the incidence of events differs significantly. We chose to perform the Cox regression analysis of the association between the CADD score and age at diagnosis, cardiac events, and sustained VT/VF separately for each cohort without any significant result for any endpoint.

A large proportion of the Nordic cohort carries the variant c.2146-1 G > C which could possibly represent a founder variant common in the Nordic countries; however, at this point, no convincing evidence for that exists. We performed a sensitivity analysis assessing all study endpoints in the study population after exclusion of carriers of this specific variant, which has not affected the risk estimated: $\mathrm{HR}_{\text {adj }} 0.998$ 95\% CI 0.942-1.057 (for sustained VT/VF), $\mathrm{HR}_{\text {adj }} 1.009$ 95\% CI 0.963-1.057 (for definite ARVC diagnosis), and $\mathrm{HR}_{\text {adj }} 1.002$ 95\% CI 0.9531.053 (for cardiac events).

\section{Study Limitations}

As ARVC diagnosis requires an extensive and dedicated diagnostic workup, it is possible that the time to diagnosis is affected by not only the intrinsic factors of the disease mechanisms but also the timing of initiation of diagnostic screening, which may be affected by multiple factors that we cannot account for. This may be particularly important for a family member who may enter the clinical follow-up program without clinical manifestation of the disease and exclusively due to the results of genetic evaluation triggered by events happening to another member of the family.

\section{Conclusions}

To the best of our knowledge, this is the first attempt to correlate the CADD score with the ARVC phenotype in carriers of $P K P 2$ variants. No correlation was found between CADD scores and clinical manifestations of ARVC, indicating that the score has no additional risk stratification value among carriers of pathogenic or likely pathogenic $P K P 2$ genetic variants.

\section{Acknowledgments}

We are grateful to the individuals involved in this study.

\section{Statement of Ethics}

Informed consent was obtained from all participants. The study by the Nordic ARVC Register is performed under approval from the Regional Ethics Committee in Lund, Sweden (2010/568 and 2017/485). The study Genetics, Mechanisms and Clinical Phenotypes of Arrhythmogenic Cardiomyopathy is approved by the University of Rochester IRB for Coordinating Center (STUDY00000523). The study was also approved by individual enrolling sites. The study complies with the Declaration of Helsinki.

\section{Conflict of Interest Statement}

The authors have no conflicts of interest to declare.

\section{Funding Sources}

This work was supported by Region Östergötland (ALF) under grant LIO-609681 and by FORSS (Medical Research Council of SoutheastSweden) undergrantFORSS/572421 andFORSS/307961. Pyotr G. Platonov is supported by The Swedish Heart-Lung Foundation and governmental funding of clinical research (ALF). Henrik K. Jensen is supported by the Novo Nordisk Foundation (NNF18OC0031258). Wojciech Zareba is supported by NIH Grant (1R01HL116906) (Mechanisms, Genotypes and Clinical Phenotypes of Arrhythmogenic Cardiomyopathy).

\section{Author Contributions}

A.S., P.P., and C.G. contributed to conception and design, and drafting the work. All authors involved in acquisition, analysis, and interpretation of data; critical revision for intellectual content; and approval of the final manuscript version.

\section{Data Availability Statement}

Due to patient confidentiality the full data set cannot be made publicly available.

\section{References}

1 Cox MG, van der Smagt JJ, Noorman M, Wiesfeld AC, Volders PG, van Langen IM, et al. Arrhythmogenic right ventricular dysplasia/ cardiomyopathy diagnostic task force criteria: impact of new task force criteria. Circ Arrhythm Electrophysiol. 2010;3(2):126-33.

2 Pilichou K, Thiene G, Bauce B, Rigato I, Lazzarini E, Migliore F, et al. Arrhythmogenic cardiomyopathy. Orphanet J Rare Dis. 2016;11:33.

3 Chen X, Bonne S, Hatzfeld M, van Roy F, Green KJ. Protein binding and functional characterization of plakophilin 2. Evidence for its diverse roles in desmosomes and beta -catenin signaling. J Biol Chem. 2002;277(12): 10512-22. 
4 Gerull B, Heuser A, Wichter T, Paul M, Basson CT, McDermott DA, et al. Mutations in the desmosomal protein plakophilin-2 are common in arrhythmogenic right ventricular cardiomyopathy. Nat Genet. 2004;36(11): 1162-4.

5 van Tintelen JP, Entius MM, Bhuiyan ZA, Jongbloed R, Wiesfeld AC, Wilde AA, et al. Plakophilin-2 mutations are the major determinant of familial arrhythmogenic right ventricular dysplasia/cardiomyopathy. Circulation. 2006;113(13):1650-8.

6 Marcus FI, McKenna WJ, Sherrill D, Basso C, Bauce B, Bluemke DA, et al. Diagnosis of arrhythmogenic right ventricular cardiomyopathy/dysplasia: proposed modification of the task force criteria. Eur Heart J. 2010;31(7): 806-14.

7 Corrado D, Wichter T, Link MS, Hauer R, Marchlinski F, Anastasakis A, et al. Treatment of arrhythmogenic right ventricular cardiomyopathy/dysplasia: an international task force consensus statement. Eur Heart J. 2015;36(46):3227-37.

8 Mazzanti A, Ng K, Faragli A, Maragna R, Chiodaroli E, Orphanou N, et al. Arrhythmogenic right ventricular cardiomyopathy: clinical course and predictors of arrhythmic risk. J Am Coll Cardiol. 2016;68(23):254050.

9 van der Pols MJ, Mast TP, Loh P, van der Heijden JF, Cramer MJ, Hauer RN, et al. Clinical characterisation and risk stratification of patients with arrhythmogenic right ventricular dysplasia/cardiomyopathy $\geq 50$ years of age. Neth Heart J. 2016;24(12):740-7.

10 Zorzi A, Rigato I, Bauce B, Pilichou K, Basso C, Thiene G, et al. Arrhythmogenic right ventricular cardiomyopathy: risk stratification and indications for defibrillator therapy. Curr Cardiol Rep. 2016;18(6):57.

11 Haugaa $\mathrm{KH}$, Bundgaard $\mathrm{H}$, Edvardsen $\mathrm{T}$, Eschen O, Gilljam T, Hansen J, et al. Management of patients with arrhythmogenic right ventricular cardiomyopathy in the Nordic countries. Scand Cardiovasc J. 2015;49(6): 299-307.

12 Towbin JA, McKenna WJ, Abrams DJ, Ackerman MJ, Calkins H, Darrieux FCC, et al. 2019 HRS expert consensus statement on evaluation, risk stratification, and management of arrhythmogenic cardiomyopathy: executive summary. Heart Rhythm. 2019;16(11):e373407.

13 Richards S, Aziz N, Bale S, Bick D, Das S, Gastier-Foster J, et al. Standards and guidelines for the interpretation of sequence variants: a joint consensus recommendation of the American College of Medical Genetics and Genomics and the Association for Molecular Pathology. Genet Med. 2015;17(5): 405-24.

14 Kircher M, Witten DM, Jain P, O'Roak BJ, Cooper GM, Shendure J. A general framework for estimating the relative pathogenicity of human genetic variants. Nat Genet. 2014; 46(3):310-5.
15 Nakagomi H, Mochizuki H, Inoue M, Hirotsu Y, Amemiya K, Sakamoto I, et al. Combined annotation-dependent depletion score for BRCA1/2 variants in patients with breast and/ or ovarian cancer. Cancer Sci. 2018;109(2): 453-61.

16 Nomura A, Tada H, Teramoto R, Konno T, Hodatsu A, Won $\mathrm{HH}$, et al. Whole exome sequencing combined with integrated variant annotation prediction identifies a causative myosin essential light chain variant in hypertrophic cardiomyopathy. J Cardiol. 2016; 67(2):133-9.

17 Borgquist R, Haugaa KH, Gilljam T, Bundgaard $\mathrm{H}$, Hansen J, Eschen $\mathrm{O}$, et al. The diagnostic performance of imaging methods in ARVC using the 2010 task force criteria. Eur Heart J Cardiovasc Imaging. 2014;15(11): 1219-25.

18 Link MS, Laidlaw D, Polonsky B, Zareba W, McNitt S, Gear K, et al. Ventricular arrhythmias in the North American multidisciplinary study of ARVC: predictors, characteristics, and treatment. J Am Coll Cardiol. 2014;64(2): $119-25$.

19 Corrado D, Wichter T, Link MS, Hauer R, Marchlinski F, Anastasakis A, et al. Treatment of arrhythmogenic right ventricular cardiomyopathy/dysplasia: an international task force consensus statement. Eur Heart J. 2015;36(46):3227-37.

20 Priori SG, Blomstrom-Lundqvist C, Mazzanti A, Blom N, Borggrefe M, Camm J, et al. 2015 ESC Guidelines for the management of patients with ventricular arrhythmias and the prevention of sudden cardiac death: the task force for the management of patients with ventricular arrhythmias and the prevention of sudden cardiac death of the European Society of Cardiology (ESC). Endorsed by: Association for European Paediatric and Congenital Cardiology (AEPC). Eur Heart J. 2015;36(41):2793867.

21 Protonotarios A, Anastasakis A, Panagiotakos DB, Antoniades L, Syrris P, Vouliotis A, et al. Arrhythmic risk assessment in genotyped families with arrhythmogenic right ventricular cardiomyopathy. Europace. 2016; 18(4):610-6.

22 Bhonsale A, Groeneweg JA, James CA, Dooijes D, Tichnell C, Jongbloed JD, et al. Impact of genotype on clinical course in arrhythmogenic right ventricular dysplasia/cardiomyopathy-associated mutation carriers. Eur Heart J. 2015;36(14):847-55.

23 Hoffman MM, Ernst J, Wilder SP, Kundaje A, Harris RS, Libbrecht $M$, et al. Integrative annotation of chromatin elements from ENCODE data. Nucleic Acids Res. 2013;41(2): $827-41$.

24 Smedley D, Schubach M, Jacobsen JOB, Köhler S, Zemojtel T, Spielmann M, et al. A whole-genome analysis framework for effective identification of pathogenic regulatory variants in mendelian disease. Am J Hum Genet. 2016;99(3):595-606.
25 Kirchner F, Schuetz A, Boldt LH, Martens K, Dittmar G, Haverkamp W, et al. Molecular insights into arrhythmogenic right ventricular cardiomyopathy caused by plakophilin-2 missense mutations. Circ Cardiovasc Genet. 2012;5(4):400-11.

26 Adachi S, Isobe M. Plakophilin-2 gene could be a causative factor in arrhythmogenic right ventricular cardiomyopathy. Circ J. 2012; 76(1):51-2

27 Jacob KA, Noorman M, Cox MG, Groeneweg JA, Hauer RN, van der Heyden MA. Geographical distribution of plakophilin-2 mutation prevalence in patients with arrhythmogenic cardiomyopathy. Neth Heart J. 2012;20(5):234-9.

28 Cerrone M, Montnach J, Lin X, Zhao YT, Zhang M, Agullo-Pascual E, et al. Plakophilin-2 is required for transcription of genes that control calcium cycling and cardiac rhythm. Nat Commun. 2017;8(1):106.

29 Bosman LP, Sammani A, James CA, CadrinTourigny J, Calkins H, van Tintelen JP, et al. Predicting arrhythmic risk in arrhythmogenic right ventricular cardiomyopathy: a systematic review and meta-analysis. Heart Rhythm. 2018;15(7):1097-107.

30 Bhonsale A, James CA, Tichnell C, Murray B, Madhavan S, Philips B, et al. Risk stratification in arrhythmogenic right ventricular dysplasia/cardiomyopathy-associated desmosomal mutation carriers. Circ Arrhythm Electrophysiol. 2013;6(3):569-78.

31 Protonotarios A, Elliott PM. Arrhythmogenic cardiomyopathy: a disease or merely a phenotype? Eur Cardiol. 2020;15:1-5.

32 Hermida A, Fressart V, Hidden-Lucet F, Donal E, Probst V, Deharo JC, et al. High risk of heart failure associated with desmoglein-2 mutations compared to plakophilin-2 mutations in arrhythmogenic right ventricular cardiomyopathy/dysplasia. Eur J Heart Fail. 2019;21(6):792-800.

33 Corrado D, Calkins H, Link MS, Leoni L, Favale S, Bevilacqua M, et al. Prophylactic implantable defibrillator in patients with arrhythmogenic right ventricular cardiomyopathy/dysplasia and no prior ventricular fibrillation or sustained ventricular tachycardia. Circulation. 2010;122(12):1144-52.

34 Cadrin-Tourigny J, Bosman LP, Nozza A, Wang W, Tadros R, Bhonsale A, et al. A new prediction model for ventricular arrhythmias in arrhythmogenic right ventricular cardiomyopathy. Eur Heart J. 2019;40(23):1850-8.

35 Cadrin-Tourigny J, Bosman LP, Wang W, Tadros R, Bhonsale A, Bourfiss M, et al. Sudden cardiac death prediction in arrhythmogenic right ventricular cardiomyopathy: a multinational collaboration. Circ Arrhythm Electrophysiol. 2021;14(1):e008509.

36 Saberniak J, Hasselberg NE, Borgquist R, Platonov PG, Sarvari SI, Smith HJ, et al. Vigorous physical activity impairs myocardial function in patients with arrhythmogenic right ventricular cardiomyopathy and in mutation positive family members. Eur J Heart Fail. 2014;16(12):1337-44. 
37 James CA, Bhonsale A, Tichnell C, Murray B, Russell SD, Tandri H, et al. Exercise increases age-related penetrance and arrhythmic risk in arrhythmogenic right ventricular dysplasia/ cardiomyopathy-associated desmosomal mutation carriers. J Am Coll Cardiol. 2013; 62(14):1290-7.

38 Lie ØH, Dejgaard LA, Saberniak J, Rootwelt C, Stokke MK, Edvardsen T, et al. Harmful effects of exercise intensity and exercise dura- tion in patients with arrhythmogenic cardiomyopathy. JACC Clin Electrophysiol. 2018; 4(6):744-53.

39 Syrris P, Ward D, Asimaki A, Sen-Chowdhry S, Ebrahim HY, Evans A, et al. Clinical expression of plakophilin-2 mutations in familial arrhythmogenic right ventricular cardiomyopathy. Circulation. 2006;113(3):356-64.

40 Svensson A, Astrom-Aneq M, Widlund KF, Fluur C, Green A, Rehnberg M, et al. Arrhyth- mogenic right ventricular cardiomyopathy: 4 Swedish families with an associated PKP2 c. 2146-1G>C variant. Am J Cardiovasc Dis. 2016;6(2):55-65.

41 Dalal D, James C, Devanagondi R, Tichnell C, Tucker A, Prakasa K, et al. Penetrance of mutations in plakophilin-2 among families with arrhythmogenic right ventricular dysplasia/ cardiomyopathy. J Am Coll Cardiol. 2006; 48(7):1416-24. 